\title{
Delannoy numbers and Legendre polytopes
}

\author{
Gábor Hetyei|| \\ Department of Mathematics and Statistics, UNC Charlotte, Charlotte, NC 28223
}

\begin{abstract}
We construct an $n$-dimensional polytope whose boundary complex is compressed and whose face numbers for any pulling triangulation are the coefficients of the powers of $(x-1) / 2$ in the $n$-th Legendre polynomial. We show that the non-central Delannoy numbers count all faces in the lexicographic pulling triangulation that contain a point in a given open quadrant. We thus provide a geometric interpretation of a relation between the central Delannoy numbers and Legendre polynomials, observed over 50 years ago. The polytopes we construct are closely related to the root polytopes introduced by Gelfand, Graev, and Postnikov.

Résumé. No construisons un polytope de dimension $n$ dont le complexe de bord est comprimé et dont les nombres de faces dans toute triangulation "en tirant des sommets" sont les coefficients des puissances de $(x-1) / 2$ dans le $n$-ième polynôme de Legendre. Nous montrons que les nombres centraux de Delannoy comptent toutes les faces dans la triangulation "en tirant des sommets" en ordre lexicographique qui contiennent un point dans un certain quadrant ouvert. Ainsi nous produisons une interprétation géometrique d'une rélation entre les nombres de Delannoy centraux et les polynômes de Legendre, notée il y a 50 ans. Nos polytopes sont reliés intimément aux polytopes de racines introduits par Gelfand, Graev, et Postnikov.
\end{abstract}

Keywords: Legendre polynomials, Delannoy numbers, root polytopes, compressed triangulations, Catalan numbers, central binomial coefficients, centrally symmetric polytopes

\section{Introduction}

The Delannoy numbers, introduced by Henri Delannoy (3) more than a hundred years ago, became recently subject of renewed interest. More than 50 years ago a somewhat mysterious connection was noted between the central Delannoy numbers $d_{n, n}$ and Legendre polynomials (6), (12), (13). Until recently, this relation was mostly dismissed as a "coincidence" (2), (16). The first interpretation was given by the present author (9) who noted that the central Delannoy numbers also form the diagonal in an asymmetric variant of the Delannoy table, where all elements are obtainable via substitution into Jacobi polynomials $P_{n}^{\alpha, \beta}(x)$, generalizing Legendre polynomials $P_{n}(x)$.

\footnotetext{
${ }^{\dagger}$ This work was supported by the NSA grant \# H98230-07-1-0073 
The subject of the present submission is a geometric interpretation of the relation between the central Delannoy numbers and the Legendre polynomials. For that purpose we construct an $n$-dimensional polytope $\mathcal{L}_{n}$ for each $n$, such that $\mathcal{L}_{n}$ has a compressed boundary complex, thus all its pulling triangulations have the same face numbers. Multiplying the number of $(j-1)$-dimensional faces by $((x-1) / 2)^{j}$ and summing over $j$ yields $P_{n}(x)$, and substituting $x=3$ gives the number of all faces. This number is also the central Delannoy number $d_{n, n}$. Our main result is that, for the lexicographic pulling triangulation of $\mathcal{L}_{n}$, the non-central Delannoy number $d_{n, n-i}$ counts the number of all faces that contain at least one point in the open quadrant defined by requiring the first $i$ coordinates to be negative.

The Legendre polytope $\mathcal{L}_{n}$ is defined as the intersection of an $n$-dimensional cross-polytope and a hyperplane in Section 3 . To prepare this definition, Section 2 explores a few facts that always hold when we consider the intersection of a centrally symmetric polytope and a hyperplane that contains the origin but no vertex. The graphs we introduce are directed generalizations of the variants of some graphs that appear in the work of Gelfand, Graev, and Postnikov (5), the key Lemma 2.4 is a generalization of a result originally due to Kapranov, Postnikov, and Zelevinski (see the first half of Lemma 12.5 in (14)).

The Legendre polytope $\mathcal{L}_{n}$ may be represented as the convex hull of the root polytope $P_{A_{n}^{+}}$and its negative. The root polytopes $P_{A_{n}^{+}}$were first studied by Gelfand, Graev and Postnikov (5). Results on these polytopes were generalized by Postnikov (14) and Wungkum Fong (4). In Section 3 we observe that some results in (5) may be restated as saying that all pulling triangulations of a root polytope that contain the origin as the least vertex, are compressed. In this form, the statement is a direct consequence of a statement of Stanley (15) and the well-known result (7) stating that the incidence matrix of every directed graph is totally unimodular. This approach easily generalizes to the directed graphs we use to model the faces in the Legendre polytope, thus we are able to show that every pulling triangulation of the boundary of the Legendre polytope that uses only the vertices, is also compressed.

We compute the face numbers of a pulling triangulation of a Legendre polytope and of a root polytope in Section 4 For the Legendre polytope this is easily done using the lexicographic order to pull the vertices, but the implied combinatorial enumeration problem does not seem to have a nice variant for root polytopes. On the other hand, the face numbers in the triangulation with respect to the revlex order may be counted with relative ease for the root polytopes and this argument has a generalization to Legendre polytopes which relies on the result for the root polytopes.

Our main result is in Section 5, where we establish the above stated geometric interpretation of the Delannoy numbers.

We hope that the approach developed here will be inspiring for researchers of generalized root polytopes, perhaps the centrally symmetric variants of those polytopes turn out to be as interesting as the Legendre polytopes. We also hope that the results enhance the interest of experts of lattice path enumeration in polyhedral face enumeration. Further suggestions may be found in the concluding Section 6 


\section{Preliminaries}

\subsection{Delannoy numbers}

The Delannoy array $\left(d_{i, j}: i, j \in \mathbb{Z}\right)$ may be defined by the recursion formula

$$
d_{i, j}=d_{i-1, j}+d_{i, j-1}+d_{i-1, j-1}
$$

with the conditions $d_{0,0}=1$ and $d_{i, j}=0$ if $i<0$ or $j<0$. Perhaps the simplest interpretation of the Delannoy number $d_{i, j}$ is that it is the number of lattice paths from $(0,0)$ to $(i, j)$ using the steps $(1,0)$, $(0,1)$ and $(1,1)$. For more interpretations see Sulanke (16), for history see Banderier and Schwer (2).

\subsection{Legendre polynomials and their connection to the Delannoy numbers}

The $n$-th Legendre polynomial $P_{n}(x)$ is the Jacobi polynomial $P_{n}^{(0,0)}(x)$, where $P_{n}^{(\alpha, \beta)}(x)=\sum_{k}\left(\begin{array}{c}n+\alpha \\ k\end{array}\right)\left(\begin{array}{c}n+\beta \\ k\end{array}\right)\left(\frac{x-1}{2}\right)^{n-k}\left(\frac{x+1}{2}\right)^{k}$. For nonnegative integer $\alpha$ we also have [Chapter 4, Exercise 15 (b)](17)

$$
\begin{gathered}
P_{n}^{(\alpha, \beta)}(x)=\sum_{j}\left(\begin{array}{c}
n+\alpha+\beta+j \\
j
\end{array}\right)\left(\begin{array}{c}
n+\alpha \\
j+\alpha
\end{array}\right)\left(\frac{x-1}{2}\right)^{j}, \quad \text { implying } \\
P_{n}(x)=\sum_{j}\left(\begin{array}{c}
n+j \\
j
\end{array}\right)\left(\begin{array}{c}
n \\
j
\end{array}\right)\left(\frac{x-1}{2}\right)^{j}
\end{gathered}
$$

It has been known for at least half a century (6), (12), (13) that $d_{n, n}=P_{n}(3)$, but no combinatorial explanation was found.

\subsection{Triangulation by pulling the vertices}

Let $\mathcal{P}$ be a polytopal complex and $L$ a set of points containing the set of vertices of $\mathcal{P}$. Let $<$ be a linear order on $L$. The pulling triangulation $\triangle_{L,<}(\mathcal{P})$ with respect to $L$ is defined recursively, as follows (1). We set $\triangle_{L,<}(\mathcal{P})=\mathcal{P}$ if $\mathcal{P}$ consists of a single vertex. Otherwise let $v_{1}$ be the least element of $L$ with respect to $<$ and set

$$
\triangle_{L,<}(\mathcal{P})=\triangle\left(\mathcal{P} \backslash v_{1}\right) \cup \bigcup_{F}\left\{\operatorname{conv}\left(\left\{v_{1}\right\} \cup G\right): G \in \triangle(\mathcal{P}(F))\right\} .
$$

Here the union runs over the facets $F$ not containing $v_{1}$ of the maximal faces of $\mathcal{P}$ which contain $v_{1}$. The complex $\mathcal{P} \backslash v_{1}$ consists of all faces of $\mathcal{P}$ not containing $v_{1}, \mathcal{P}(F)$ consists of all faces of $\mathcal{P}$ contained in $F$. The triangulations $\triangle\left(\mathcal{P} \backslash v_{1}\right)$ and $\triangle(\mathcal{P}(F))$ are with respect to $L \backslash\left\{v_{1}\right\}$ and the restriction of $<$. In the 
event when $L$ equals the vertex set of $\mathcal{P}$ we will write $\triangle_{<}$instead of $\triangle_{L,<}$ and refer to the triangulation as a pulling triangulation that uses only the vertices of $\mathcal{P}$.

Counting the faces of $\triangle_{L,<}(\mathcal{P})$ is hard in general. In one important special case, however, it is easy, thanks to Stanley (15, Example 2.4 (a)). Assume that $\mathcal{P}$ is the face complex of a polytope $P$ whose vertices have integer coordinates and let $L=V(P)$. The order $<$ is compressed if all facets in $\triangle_{<}(P)$ have the same minimal volume. The face numbers of $\triangle_{<}(P)$ are the same for any compressed order $<$.

Proposition 1.1 (Stanley) Suppose that one of the vertices of $P$ is the origin and that the matrix whose rows are the vertices of $P$ is totally unimodular. Let $<$ be any ordering on $V(P)$ such that the origin is the least vertex with respect to $V(P)$. Then $<$ is compressed.

\section{Central sections of centrally symmetric polytopes}

Let $P \subset \mathbb{R}^{n}$ be a centrally symmetric polytope, centered at the origin 0 . Let $H$ be a hyperplane containing 0 but no vertex of $P$. We call $Q:=P \cap H$ a non-degenerate central section of $P$.

Assume that $H$ is given by $\sum_{i=1}^{n} \lambda_{i} x_{i}=\langle\lambda \mid x\rangle=0$. The vertex set $V(P)$ of $P$ may then be partitioned into two disjoint sets $V_{+}(P):=\{x \in V(P):\langle\lambda \mid x\rangle>0\}$ and $V_{-}(P):=\{x \in V(P)$ : $\langle\lambda \mid x\rangle<0\}$. Each vertex of $Q$ is of the form $H \cap[u,-v]$ where $[u,-v]$ is the line segment connecting $u \in V_{+}(P)$ and $-v \in V_{-}(P)$ (thus $v \in V_{+}(P)$ ). Obviously, $[u,-v]$ must be an edge of $P$. We may thus represent the vertex set of $Q=P \cap H$ by a graph $G=G(P, H)$ on the vertex set $V(G):=V_{+}(P)$ and letting $(u, v)$ be a directed edge in $G$ exactly when $[u,-v] \cap H$ is a vertex of $Q$. Note that $G(P, H)$ contains no loops. Since no two edges can intersect except at the vertices, we obtain:

Lemma 2.1 Each vertex of $Q$ is represented by a unique edge $(u, v)$ in $G(P, H)$.

Definition 2.2 Let $G$ be a directed graph on the vertex set $V(G)$, edge set $E(G)$ with no multiple edges. Let $S$ and $T$ be disjoint subsets of $V$. The directed restriction of $G$ to $(S, T)$ is the digraph with vertex set $S \cup T$ with edge set $\{(s, t) \in E(G): s \in S, t \in T\}$.

Each face of $Q$ is of the form $F \cap H$ where $F$ is a face of $P$. Obviously, the set of vertices $V(F)$ of $F$ is a subset of $V(P)$. Introducing $V_{+}(F):=V_{+}(P) \cap V(F)$ and $V_{-}(F):=V_{-}(P) \cap V(F)$, each vertex of $F \cap H$ is of the form $[u,-v] \cap H$ where $u \in V_{+}(F)$ and $-v \in V_{-}(F)$. Since the set of vertices $F \cap H$ is a subset of the set of vertices of $Q$, each vertex $[u,-v] \cap H$ of $F \cap H$ must satisfy that $(u, v)$ is an edge of $G(P, H)$. Conversely, if $(u, v)$ is an edge in $G(P, H), u \in V_{+}(F)$ and $-v \in V_{-}(F)$ then the segment $[u,-v]$ is a subset of $F$ and $[u,-v] \cap H$ is a vertex in $F \cap H$. Thus we obtain:

Proposition 2.3 Assume the vertices of $Q=P \cap H$ are represented by the edges of the graph $G=$ $G(P, H)$. Given a face $F$ of $P$, the vertices contained in the face $F \cap H$ of $Q$ are represented by the edges in the directed restriction of $G(P, H)$ to $\left(V_{+}(F),-V_{-}(F)\right)$. 
Assume from now on that $P$ is simplicial. Then, for each face $F \subset P$ the directed restriction of $G(P, H)$ to $\left(V_{+}(F),-V_{-}(F)\right)$ is a complete bipartite graph, with each edge directed towards its endpoint in $V_{-}(F)$. The following Lemma is a generalization of a result of Kapranov, Postnikov, and Zelevinski (see the first half of Lemma 12.5 in (14)).

Lemma 2.4 Let $F \subset P$ be a face of $P$. A subset $S$ of the edges of the directed restriction of $G(P, H)$ to $\left(V_{+}(F),-V_{-}(F)\right)$ represents an affinely independent set of vertices if and only if, disregarding the orientation of the edges, the set $S$ contains no circuit.

\section{Legendre polytopes and root polytopes}

The standard cross-polytope $\mathcal{O}_{n+1} \subset \mathbb{R}^{n+1}$ is the convex hull of $\left\{e_{0},-e_{0}, \ldots, e_{n},-e_{n}\right\}$ where the $e_{i}$ 's are the standard basis of $\mathbb{R}^{n+1}$. (We start indexing from 0 , for technical reasons.) Let $2 \mathcal{O}_{n+1}$ be the polytope obtained from $\mathcal{O}_{n+1}$ by applying a dilation of a factor of 2 , centered at $\mathbf{0}$.

Definition 3.1 We define the Legendre polytope $\mathcal{L}_{n}$ as the non-degenerate central section of $2 \mathcal{O}_{n+1}$ with the hyperplane $H_{n}:=\left\{\left(x_{0}, \ldots, x_{n}\right): \sum_{i=0}^{n} x_{i}=0\right\}$.

The vertices of $\mathcal{L}_{n}$ are all points of the form $e_{i}-e_{j}$ where $i \neq j$. We may encode each vertex of $\mathcal{L}_{n}$ by an edge $\left(e_{i}, e_{j}\right)$. The graph $G\left(\mathcal{L}_{n}, H_{n}\right)$ is then the complete digraph on the vertex set $\left\{e_{0}, \ldots, e_{n}\right\}$, containing all directed edges $\left(e_{i}, e_{j}\right)$ where $i \neq j$. Each proper face of $\mathcal{L}_{n}$ is of the form $F \cap H_{n}$ where $F$ is a face of $2 \mathcal{O}_{n+1}$. A subset of $\left\{2 e_{0},-2 e_{0}, \ldots, 2 e_{n},-2 e_{n}\right\}$ is the vertex set of a face $F$ of $2 \mathcal{O}_{n+1}$ exactly when it does not contain both $2 e_{i}$ and $-2 e_{i}$ for some $i$. In particular, each facet of $2 \mathcal{O}_{n+1}$ is of the form $\operatorname{conv}\left\{\varepsilon_{0} 2 e_{0}, \ldots, \varepsilon_{n} 2 e_{n}\right\}$ where $\varepsilon_{0}, \ldots, \varepsilon_{n} \in\{1,-1\}$. Thus we may observe the following.

Lemma $3.2 A$ set $S \subset\left\{\left(e_{i}, e_{j}\right): i \neq j\right\}$ of edges represents all vertices in a facet of the boundary $\partial \mathcal{L}_{n}$ of $\mathcal{L}_{n}$ if and only if there is a proper subset $A$ of $A \subset\left\{e_{0}, \ldots, e_{n}\right\}$ such that $S$ consists of all edges starting in $A$ and ending in $\left\{e_{0}, \ldots, e_{n}\right\} \backslash A$.

This lemma may be rephrased in terms of admissibility, originally introduced in (5) for root polytopes.

Definition 3.3 We call a set $S$ of edges in a directed graph $G$ on the vertex set admissible if there is no vertex $v$ such that both $(u, v) \in G(v, w) \in G$ hold for some vertices $u$ and $w$.

Lemma 3.2 is equivalent to the following.

Lemma 3.4 A set $S \subset\left\{\left(e_{i}, e_{j}\right): i \neq j\right\}$ of edges represents a subset of the vertex set of a face in $\partial \mathcal{L}_{n}$ if and only if it is admissible. 
The convex hull of $\mathbf{0}$ and the vertex set $\left\{e_{i}-e_{j}: i<j\right\}$ is the root polytope $P_{A_{n}^{+}}$, first studied by Gelfand, Graev and Postnikov (5). We may think of $\mathcal{L}_{n}$ as the convex hull of $P_{A_{n}^{+}}$and $-P_{A_{n}^{+}}$. Let us review now two triangulations of the root polytope $P_{A_{n}^{+}}$, introduced in (5). The elements of $V\left(P_{A_{n}^{+}}\right) \backslash\{\mathbf{0}\}$ correspond to the directed edges $\left(e_{i}, e_{j}\right)$ where $i<j$. Since all edges are directed from the lower indexed edge towards the higher indexed edge, we could omit indicating the orientation and use undirected edges, and use $(i, j)$ as a shorthand for $\left(e_{i}, e_{j}\right)$. We would then arrive exactly at the notation used in (5). All facets of $P_{A_{n}^{+}}$contain $\mathbf{0}$ since $P_{A_{n}^{+}}$is a cone over $\mathbf{0}$. The faces of $P_{A_{n}^{+}}$that do not contain $\mathbf{0}$ are subsets of $\partial \mathcal{L}_{n}$, their union is $P_{A_{n}^{+}} \cap \partial \mathcal{L}_{n}$. By Lemma 3.4, a set of vertices contained in a face of $\mathcal{L}_{n}$ must be represented by an admissible set of edges, and here our notion of admissibility specializes to the definition given in (5).

As seen in Lemma 2.4 (or Lemma 12.5 in (14)) a set of vertices contained in a face of $P_{A_{n}^{+}} \cap \partial \mathcal{L}_{n}$ is affinely independent if and only if the associated edges form no cycle. In particular, facets in any triangulation of $P_{A_{n}^{+}}$are associated with admissible trees on the vertex set $\left\{e_{0}, \ldots, e_{n}\right\}$. (The vertices represented by the tree generate a facet in a triangulation of $P_{A_{n}^{+}} \cap \partial \mathcal{L}_{n}$, coning over 0 results in a triangulation of $P_{A_{n}^{+}}$.) There are two triangulations of $P_{A_{n}^{+}} \cap \partial \mathcal{L}_{n}$ explicitly given in (5): the standard and the anti-standard triangulation. The standard triangulation is associated to the set of all admissible trees having no intersections: the edges $\left(e_{i}, e_{j}\right)$ and $\left(e_{k}, e_{l}\right)$ are intersecting if $i<k<j<l$ holds. The anti-standard triangulation is associated to the set of all admissible trees having no enclosed edges: $\left(e_{i}, e_{j}\right)$ and $\left(e_{k}, e_{l}\right)$ are enclosed if $i<k<l<j$ holds.

Both the standard and anti-standard triangulations are pulling triangulations of $P_{A_{n}^{+}}$.

Definition 3.5 The revlex order on $V\left(P_{A_{n}^{+}}\right) \backslash\{0\}$ is defined by setting $\left(e_{i}, e_{j}\right)<\left(e_{k}, e_{l}\right)$ if $j<l$ or $j=l$ and $i>k$. The lexicographic order on $V\left(P_{A_{n}^{+}}\right) \backslash\{\mathbf{0}\}$ is defined by setting $\left(e_{i}, e_{j}\right)<\left(e_{k}, e_{l}\right)$ if $i<k$ or $i=k$ and $j<l$.

Lemma 3.6 The standard triangulation of $P_{A_{n}^{+}}$is the pulling triangulation with $L=V\left(P_{A_{n}^{+}}\right)$and the revlex order on $V\left(P_{A_{n}^{+}}\right) \backslash\{\mathbf{0}\}$ extended to $V\left(P_{A_{n}^{+}}\right)$by making $\mathbf{0}$ the least element.

Lemma 3.7 The anti-standard triangulation of $P_{A_{n}^{+}}$is the pulling triangulation with $L=V\left(P_{A_{n}^{+}}\right)$and the lex order on $V\left(P_{A_{n}^{+}}\right) \backslash\{\mathbf{0}\}$ extended to $V\left(P_{A_{n}^{+}}\right)$by making $\mathbf{0}$ the least element.

It was observed in (5) that the facets in the standard and anti-standard triangulations have all the same minimal volume. More generally, (5, Lemma 5.3) may be rephrased as

Proposition 3.8 (Gelfand-Graev-Postnikov) Any pulling triangulation $\triangle_{L,<}\left(P_{A_{n}^{+}}\right)$of $P_{A_{n}^{+}}$satisfying $L=V\left(P_{A_{n}^{+}}\right)$and having $\mathbf{0}$ as the least element in the order, is compressed.

Proposition 3.8 is also an easy consequence of Proposition 1.1 and the following result (7), (8).

Theorem 3.9 (Heller) The incidence matrix of a directed graph is totally unimodular. 
This proof extends easily to a similar statement on $\mathcal{L}_{n}$.

Theorem 3.10 Any pulling triangulation $\triangle_{L,<}\left(\mathcal{L}_{n}\right)$ of $\mathcal{L}_{n}$ satisfying $L=V\left(\mathcal{L}_{n}\right) \cup\{\mathbf{0}\}$ and having $\mathbf{0}$ as the least element in the order, is compressed.

Since pulling the interior point $\mathbf{0}$ results in coning over a pulling triangulation of $\partial \mathcal{L}_{n}$, we obtain:

Corollary 3.11 All pulling triangulations of $\partial \mathcal{L}_{n}$ (using only the vertices) have the same face numbers.

\section{The $F$-polynomials of the pulling triangulations}

Let us compute now the face numbers in any pulling triangulation $\triangle_{<}\left(\partial \mathcal{L}_{n}\right)$ of $\partial \mathcal{L}_{n}$ that uses only the vertices. By Corollary 3.11 we only need to find these numbers for one linear order $<$. The easiest seems to use the lexicographic order: we set $\left(e_{i}, e_{j}\right)<\left(e_{k}, e_{l}\right)$ if $i<k$ or $i=k$ and $j<l$. In the previous section, this order was considered for the vertices of the root polytope $P_{A_{n}^{+}}$only, now we extend it to all vertices of the Legendre polytope $\mathcal{L}_{n}$.

Lemma 4.1 A set of vertices $\left\{\left(e_{s_{1}}, e_{t_{1}}\right),\left(e_{s_{2}}, e_{t_{2}}\right), \ldots,\left(e_{s_{j}}, e_{t_{j}}\right)\right\}$, satisfying $\left(e_{s_{1}}, e_{t_{1}}\right)<\cdots<\left(e_{s_{j}}, e_{t_{j}}\right)$ in the lexicographic order is a face of $\triangle_{<}\left(\partial \mathcal{L}_{n}\right)$ if and only if the following holds:

(i) the sets $\left\{s_{1}, \ldots, s_{j}\right\}$ and $\left\{t_{1}, \ldots, t_{j}\right\}$ are disjoint;

(ii) we have $t_{1} \leq \cdots \leq t_{j}$.

Theorem 4.2 The number of $(j-1)$-dimensional faces in any pulling triangulation of $\partial \mathcal{L}_{n}$ that uses only the vertices of $\mathcal{L}_{n}$ is

$$
f_{j-1}\left(\triangle_{<}\left(\partial \mathcal{L}_{n}\right)\right)=\left(\begin{array}{c}
n+j \\
j
\end{array}\right)\left(\begin{array}{l}
n \\
j
\end{array}\right)
$$

Proof: We may assume $<$ is the lexicographic order. Using Lemma 4.1 we may show

$$
f_{j-1}\left(\triangle_{<}\left(\partial \mathcal{L}_{n}\right)\right)=\sum_{u, v}\left(\begin{array}{c}
n+1 \\
u, v, n+1-u-v
\end{array}\right)\left(\begin{array}{c}
j-1 \\
j-u, j-v, u+v-j-1
\end{array}\right)
$$

The sum on the right hand side simplifies to $\left(\begin{array}{c}n+j \\ j, j, n-j\end{array}\right)=\left(\begin{array}{c}n+j \\ j\end{array}\right)\left(\begin{array}{c}n \\ j\end{array}\right)$.

Let us introduce now the $F$-polynomial of a simplicial complex $\triangle$ as $F_{\triangle}(x):=\sum_{j=0}^{d} f_{j-1}\left(\frac{x-1}{2}\right)^{j}$. Here $d-1=\operatorname{dim}(\triangle)$ and $f_{j-1}$ is the number of $(j-1)$-dimensional faces. This polynomial, a variant of the usual $f$-polynomial, was shown to be related to certain orthogonal polynomials for the order complexes of some spherical posets in (10) and for a triangulation in (11). 
Corollary 4.3 The F-polynomial of any pulling any pulling triangulation of $\partial \mathcal{L}_{n}$ that uses only the vertices of $\mathcal{L}_{n}$ is $P_{n}(x)$, the $n$-th Legendre polynomial.

We conclude this section by computing the face numbers in any pulling triangulation of $P_{A_{n}^{+}} \cap \partial \mathcal{L}_{n}$ that uses only the vertices. The pulling triangulations of the root polytope $P_{A_{n}^{+}}$whose least element is $\mathbf{0}$ are obtained from the pulling triangulations of $P_{A_{n}^{+}} \cap \partial \mathcal{L}_{n}$ via coning over 0 . At the level of the $F$-polynomials, coning over a single vertex induces multiplication by a factor of $(x+1) / 2$.

Theorem 4.4 The number of $(j-1)$-dimensional faces in any pulling triangulation of $P_{A_{n}^{+}} \cap \partial \mathcal{L}_{n}$ that uses only the vertices is

$$
f_{j-1}\left(\triangle_{<}\left(P_{A_{n}^{+}} \cap \partial \mathcal{L}_{n}\right)\right)=\frac{1}{j+1}\left(\begin{array}{c}
n+j \\
j
\end{array}\right)\left(\begin{array}{c}
n \\
j
\end{array}\right) .
$$

Proof: Now we assume that we are dealing with the revlex order. Introducing $f(n, j)$ as a shorthand for $f_{j-1}\left(\triangle_{<}\left(P_{A_{n}^{+}} \cap \partial \mathcal{L}_{n}\right)\right)$, we have the following recursion formula.

$$
f(n, j)=\sum_{0 \leq u<v \leq n} \sum_{k=0}^{j-1} f(v-u-1, k) \cdot f(n-v, j-k-1) .
$$

Here $\left(e_{u}, e_{v}\right)$ represents the least vertex in a face. The initial condition on the numbers $f(n, j)$ is $f(n, 0)=$ 1 , easy substitution into 5 ) gives $f(n, 1)=\left(\begin{array}{c}n+1 \\ 2\end{array}\right)$. We may use the recursion formula $(5)$ to prove by induction on $j$ that $f(n, j)$ is of the form $C_{j}\left(\begin{array}{c}n+j \\ 2 j\end{array}\right)$ where the number $C_{j}$ does not depend on $n$. In fact, the statement is true for $j=0$ and $j=1$ (with $C_{0}=1$ and $C_{1}=1$ ), and the induction step is the following:

$$
\begin{aligned}
f(n, j)= & \sum_{0 \leq u<v \leq n} \sum_{k=0}^{j-1} C_{k}\left(\begin{array}{c}
v-u-1+k \\
2 k
\end{array}\right) \cdot C_{j-k-1}\left(\begin{array}{c}
n-v+j-k-1 \\
2 j-2 k-2
\end{array}\right) \\
= & \sum_{k=0}^{j-1} C_{k} C_{j-k-1} \sum_{0 \leq u<v \leq n}\left(\begin{array}{c}
v-u-1+k \\
2 k
\end{array}\right)\left(\begin{array}{c}
n-v+j-k-1 \\
2 j-2 k-2
\end{array}\right) \\
= & \sum_{k=0}^{j-1} C_{k} C_{j-k-1}\left(\begin{array}{c}
n+j \\
2 j
\end{array}\right) .
\end{aligned}
$$

Thus we have also shown that the numbers $C_{j}$ satisfy the recursion formula $C_{j}=\sum_{k=0}^{j-1} C_{k} C_{j-k-1}$. This, together with $C_{0}=C_{1}=1$ implies that $C_{j}$ is the $j$-th Catalan number, and we have

$$
f(n, j)=\frac{1}{j+1}\left(\begin{array}{c}
2 j \\
j
\end{array}\right)\left(\begin{array}{c}
n+j \\
2 j
\end{array}\right)=\frac{1}{j+1}\left(\begin{array}{c}
n+j \\
j
\end{array}\right)\left(\begin{array}{l}
n \\
j
\end{array}\right) .
$$


It should be noted that Theorem 4.2 also has a proof, analogous to the proof of Theorem 4.4 that relies on the following extension of the revlex order to the vertex set of $\mathcal{L}_{n}$ : we set $\left(e_{i}, e_{j}\right)<\left(e_{k}, e_{l}\right)$ if one of the following holds:

(i) $\max (i, j)<\max (k, l)$;

(ii) $\max (i, j)=\max (k, l)$ and $\min (i, j)>\min (k, l)$;

(iii) $\{i, j\}=\{k, l\}$ and $i<j$ and $k>l$.

Introducing $g(n, j)$ as a shorthand for $f_{j-1}\left(\partial \mathcal{L}_{n}\right)$, an argument similar to the one in the proof of Theorem 4.4 yields the recursion formula

$$
g(n, j)=2 \sum_{0 \leq u<v \leq n} \sum_{k=0}^{j-1} f(v-u-1, k) g(n-v, j-k-1),
$$

where the numbers $g(n, j)$ are of the form $B_{j}\left(\begin{array}{c}n+j \\ 2 j\end{array}\right)$, and $B_{j}$ is the central binomial coefficient $\left(\begin{array}{c}2 j \\ j\end{array}\right)$.

Remark 4.5 The number of facets in any pulling triangulation of $P_{A_{n}^{+}} \cap \partial \mathcal{L}_{n}$ is the Catalan number $C_{n}$, as it was already stated in (5). As a consequence of Theorem 4.2, the number of facets in any pulling triangulation of $\partial \mathcal{L}_{n}$ is the central binomial coefficient $\left(\begin{array}{c}2 n \\ n\end{array}\right)$. The relation between the root polytope $P_{A_{n}^{+}}$ and the Legendre polytope $\mathcal{L}_{n}$ is thus a "geometric enhancement" of the relation between the Catalan numbers and central binomial coefficients, and this undercurrent seems especially highlighted by the use of the revlex order. It is unknown to the present author, whether the lex order could also be used efficiently to establish such a connection.

In analogy to Corollary 4.3 we have the following consequence of Theorem 4.4

Corollary 4.6 The F-polynomial of any pulling triangulation of $P_{A_{n}^{+}} \cap \partial \mathcal{L}_{n}$ that uses only the vertices is $P_{n}^{(1,-1)}(x) /(n+1)$. Here $P_{n}^{(1,-1)}(x)$ is a Jacobi polynomial.

In fact, direct substitution of the numbers in Theorem 4.4 into the definition of the $F$-polynomial gives $\sum_{j=0}^{n} \frac{1}{j+1}\left(\begin{array}{c}n+j \\ j\end{array}\right)\left(\begin{array}{c}n \\ j\end{array}\right)\left(\frac{x-1}{2}\right)^{j}$, which may be rewritten as $\frac{1}{n+1} \sum_{j=0}^{n}\left(\begin{array}{c}n+j \\ j\end{array}\right)\left(\begin{array}{c}n+1 \\ j+1\end{array}\right)\left(\frac{x-1}{2}\right)^{j}$. This expression is $P_{n}^{(1,-1)}(x) /(n+1)$, by 2 .

\section{Delannoy numbers and quadrants in the Legendre polytope}

Since the Delannoy number $d_{n, n-i}$ enumerates the number of lattice paths from $(0,0)$ to $(n, n-i)$, using only steps $(1,0),(0,1)$ and $(1,1)$, the following equality is obvious:

$$
d_{n, n-i}=\sum_{k=0}^{n-i}\left(\begin{array}{c}
n+k \\
n-i-k, k+i, k
\end{array}\right)=\sum_{k=0}^{n-i}\left(\begin{array}{c}
n+k \\
k+i
\end{array}\right)\left(\begin{array}{c}
n-i \\
k
\end{array}\right)
$$


Inspired by equation 4 , let us rewrite $\left(\begin{array}{c}n+k \\ k+i\end{array}\right)\left(\begin{array}{c}n-i \\ k\end{array}\right)$ as follows:

$$
\begin{aligned}
\left(\begin{array}{c}
n+k \\
k+i
\end{array}\right)\left(\begin{array}{c}
n-i \\
k
\end{array}\right)= & \sum_{u=1}^{n-i+1}\left(\begin{array}{c}
n-i+1 \\
u
\end{array}\right)\left(\begin{array}{l}
k+i-1 \\
k+i-u
\end{array}\right) \sum_{v}\left(\begin{array}{c}
n-i+1-u \\
v
\end{array}\right)\left(\begin{array}{l}
u-1 \\
k-v
\end{array}\right) \\
& =\sum_{u, v}\left(\begin{array}{c}
n-i+1 \\
u, v+n-i-1
\end{array}\right)\left(\begin{array}{c}
k-u-v \\
k+i-u, k-v, u+v-k-1
\end{array}\right) .
\end{aligned}
$$

Using the above formula we may observe that $\left(\begin{array}{c}n+k \\ k+i\end{array}\right)\left(\begin{array}{c}n-i \\ k\end{array}\right)$ is the number of ways to perform the following procedure:

1. For some $u$ and $v$, select an $u$-element subset $U$ and a $v$-element subset $V$ of $\{i, i+1, \ldots, n\}$ such that $U$ and $V$ are disjoint $\left(\left(\begin{array}{c}n-i+1 \\ u, v, n-i+1-u-v\end{array}\right)\right.$ ways).

2. Add $\{0,1, \ldots, i-1\}$ to $V$, and select $s_{1} \leq \ldots \leq s_{k+i}$ and $t_{1} \leq \ldots \leq t_{k+i}$ such that $\left\{s_{1}, \ldots, s_{k+i}\right\}=U,\left\{t_{1}, \ldots, t_{k+i}\right\}=V \cup\{0,1, \ldots, i-1\}$, and $\left(e_{s_{1}}, e_{t_{1}}\right)<\cdots<\left(e_{s_{k+i}}, e_{t_{k+i}}\right)$ is a strictly increasing chain in the lexicographic order.

The second phase may be performed in $\left(\begin{array}{c}k+i-1 \\ k+i-u, k-v, u+v-k-1\end{array}\right)$ ways since it will happen $k-v$ times that we increase only the first coordinate, $k+i-u$ times that we only increase the second coordinate, and $u+v-k-1$ times that we increase both coordinates. In analogy to Theorem 4.2 we obtained the following result.

Theorem 5.1 The number of those ( $k+i-1)$-dimensional faces in the lexicographic pulling triangulation of $\partial \mathcal{L}_{n}$ (using only the vertices of $\mathcal{L}_{n}$ ) which contain at least one vertex of the form $e_{s}-e_{t}$ for each $t \in\{0,1, \ldots, i-1\}$ is $\left(\begin{array}{c}n+k \\ k+i\end{array}\right)\left(\begin{array}{c}n-i \\ k\end{array}\right)$.

Note that this theorem includes Theorem 4.2 as the special case $i=0$. However, we have this generalization for the lexicographic pulling triangulation only. As a consequence of (6) and Theorem 5.1 we have the following result.

Corollary 5.2 The Delannoy number $d_{n, n-i}$ is the number of all those faces in the lexicographic pulling triangulation of $\partial \mathcal{L}_{n}$ which contain at least one vertex of the form $e_{s}-e_{t}$ for each $t \in\{0,1, \ldots, i-1\}$.

In particular, $d_{n, n}$ is the number of all faces in the lexicographic pulling triangulation of $\partial \mathcal{L}_{n}$ and thus equal to $P_{n}(3)$, by Corollary 4.3 .

Corollary 5.2 has the following geometric interpretation.

Theorem 5.3 For $i>0$ the Delannoy number $d_{n, n-i}$ is the number of all faces $F$ in the lexicographic pulling triangulation of $\partial \mathcal{L}_{n}$ that contain at least one point in the quadrant $\left\{\left(x_{0}, \ldots, x_{n}\right): x_{0}<0, x_{1}<\right.$ $\left.0, \ldots, x_{i}<0\right\}$. 
Proof: A face in $\triangle_{<}\left(\partial \mathcal{L}_{n}\right)$ contains at least one vertex of the form $e_{s}-e_{t}$ for each $t \in\{0,1, \ldots, i-1\}$ if and only if it contains a point $\left(x_{0}, \ldots, x_{n}\right)$ satisfying $x_{0}<0, x_{1}<0, \ldots, x_{i-1}<0$. In fact, if there exists vertices of the form $e_{s_{0}}-e_{0}, e_{s_{1}}-e_{1}, \ldots, e_{s_{i-1}}-e_{i-1}$ then the coordinates $\left(x_{0}, \ldots, x_{n}\right)$ of the point $\frac{1}{i} \sum_{j=0}^{i-1}\left(e_{s_{j}}-e_{j}\right)$ satisfy $x_{0}<0, x_{1}<0, \ldots, x_{i-1}<0$. Conversely, if there is a point $\left(x_{0}, \ldots, x_{n}\right)$ satisfying $x_{0}<0, x_{1}<0, \ldots, x_{i-1}<0$ in the convex hull, then for each $j \leq i-1$ the inequality $x_{j}<0$ forces the existence of a vertex $\left(e_{s_{j}}-e_{j}\right)$ in the triangulation.

Theorem 5.3 motivates calling the quadrants $\left\{\left(x_{0}, \ldots, x_{n}\right): x_{0}<0, x_{1}<0, \ldots, x_{i}<0\right\} \cap \mathcal{L}_{n}$ the Delannoy quadrants of the Legendre polytope.

\section{Concluding remarks}

Fundamentally, there are two ways to define a polytope: as a convex hull of vertices, or as an intersection of half-spaces and hyperplanes. It appears that the root polytopes introduced by Gelfand, Graev, and Postnikov (5) are most easily generalized in terms of the first approach: one replaces the set of vertices with a set that has a more complicated geometry, but still many symmetries. Legendre polytopes, however, are very naturally generalized in terms of the second approach, as indicated in Section 2 It seems reasonable to expect that non-degenerate central sections of other centrally symmetric polytopes will have interesting geometric and combinatorial properties. Lemma 2.4 indicates that the digraph generalization of the graphical approach that can be found in (5) is suitable to visualize the face structure of any non-degenerate central section of any simplicial centrally symmetric polytope.

In our work it was convenient to know that face enumeration in a pulling triangulation does not depend on the order of vertices by Stanley's example (15) and a well-known unimodularity result. The question naturally arises: under which conditions can we guarantee that a non-degenerate central section of a centrally symmetric polytope is compressed? Here the term "compressed" seems to be most naturally defined in terms of considering all pulling triangulations that use the vertices, $\mathbf{0}$, and make $\mathbf{0}$ the least point in the order. In particular, is it true that, subject to assumptions, a non-degenerate central section of a compressed centrally symmetric polytope is compressed?

Our main result on the Delannoy numbers counting certain faces in the Legendre polytope applies to the lexicographic pulling triangulation only. It seems worth exploring whether an analogous result exist for the revlex pulling triangulation, or whether there is a result that can be stated independently of the order on the vertices.

\section{Acknowledgements}

I wish to thank Alexander Postnikov and Richard Stanley, for valuable background information on root polytopes. This work was supported by the NSA grant \# H98230-07-1-0073. 


\section{References}

[1] C. Athanasiadis, $h^{*}$-Vectors, Eulerian Polynomials and Stable Polytopes of Graphs, Electron. J. Combin. Vol. 11 (2) (2004), \# R6, 13 pp.

[2] C. Banderier and S. Schwer, Why Delannoy numbers ?, preprint 2004, http: / / xxx.lanl.gov/abs/math/0411128, to appear in J. Statist. Plan. Inference.

[3] H. Delannoy, Employ d'échiquier pour la résolution de certains problèmes de probabilités, Assoc. Franc. Bordeaux 24 (1895), 70-90.

[4] Wungkum Fong, Triangulations and combinatorial properties of convex polytopes, PhD. Thesis, MIT, 2000.

[5] I. M. Gelfand, M. I. Graev, A. Postnikov, Combinatorics of hypergeometric functions associated with positive roots, in Arnold-Gelfand Mathematical Seminars: Geometry and Singularity Theory, Birkhauser, Boston, 1996, 205-221.

[6] I.J. Good, Legendre polynomials and trinomial random walks, Proc. Cambridge Philos. Soc. 54 (1958), 39-42.

[7] I. Heller, On linear systems with integral valued solutions, Pacific J. Math 7 (1957), 1351-1364.

[8] I. Heller and A. J. Hoffman, On unimodular matrices, Pacific J. Math 4 (1962), 1321-1327.

[9] G. Hetyei, Central Delannoy numbers and balanced Cohen-Macaulay complexes, Ann. Comb. 10 (2006) 443-462.

[10] G. Hetyei, Orthogonal polynomials represented by CW-spheres, Electron. J. Combin. 11(2) (2004), \#R4, 28 pp.

[11] G. Hetyei, Tchebyshev triangulations of stable simplicial complexes, to appear in J. Combin. Theory. Ser. A.

[12] D.F. Lawden, On the solution of a linear difference equation, Mah. Gaz. 36 (1952), 193-196.

[13] L. Moser and W. Zayachkowski, Lattice paths with diagonal steps, Scripta Math. 26 (1963), 223229.

[14] A. Postnikov, Permutohedra, Associahedra, and Beyond, preprint 2005, arXiv: math.CO/0507163.

[15] R. P. Stanley, Decompositions of rational convex polytopes, Annals of Discrete Mathematics 6 (1980), 333-342.

[16] R.A. Sulanke, Objects counted by the central Delannoy numbers, J. Integer Seq. 6 (2003), Article $03.1 .5,19 \mathrm{pp}$.

[17] H.S. Wilf, "generatingfunctionology," Academic Press, 1994. 